\title{
Functional micro-structured optical fibers
}

\section{Yong Xu, Yanyi Huang, Amnon Yariv}

Yong Xu, Yanyi Huang, Amnon Yariv, "Functional micro-structured optical fibers," Proc. SPIE 5733, Photonic Crystal Materials and Devices III, (13 April 2005); doi: $10.1117 / 12.611504$

Event: Integrated Optoelectronic Devices 2005, 2005, San Jose, California, United States 


\title{
Functional micro-structured optical fibers
}

\author{
Yong $\mathrm{Xu}^{*}$, Yanyi Huang, and Amnon Yariv, \\ Department of Applied Physics, MS 128-95, \\ California Institute of Technology, \\ Pasadena, CA 91125, USA.
}

\begin{abstract}
We developed a procedure for the fabrication of functional micro-structured optical fibers (MOFs) through a selective filling technique. In this method, we selectively infiltrate the conventional silica MOF with a UV curable polymer and construct a mold for the fabrication of the functional MOF. Subsequently, we can build the functional MOF by introducing novel materials such as nonlinear optical polymers, nano-structures, and water-based solutions. Such functional MOFs can find wide applications in areas that include nonlinear optics, spectroscopy, chemical and biological sensing.
\end{abstract}

Keywords: Micro-structured fibers, nonlinear optics, polymer, microfluidic optics, spectroscopy.

\section{INTRODUCTION}

Recently, micro-structured optical fibers (MOFs), which typically consist of two dielectric materials with a large refractive index difference, have been extensively investigated. ${ }^{1-9}$ Due to the large index contrast, a properly designed MOF can produce strong Bragg reflection and lead to very effective optical confinement in a low index core (air for example). ${ }^{1}$ Since low index core guiding can not be achieved in the conventional fiber geometry, where optical guiding is provided by total internal reflection, research on hollow core MOF has led to many interested discoveries. ${ }^{1-5}$ Additionally, solid core MOFs have also been proposed and demonstrated. ${ }^{6}$ Since the cladding structure of a solid core MOF is typically composed of a high index material (such as silica) and a low index material (such as air), the composite cladding has an effective refractive index lower than that of the central core region. As a result, in the solid core MOF light can be confined within the high index core due to effective index guiding. ${ }^{5}$ In this regard, the confinement mechanism of a solid core MOF is the same as that of the conventional step index fiber. However, since the effective cladding index of the solid core MOF can be controlled by changing the geometrical composition of the cladding structure, the solid core MOF offers more flexibility and freedom in the realization of total internal reflection guiding as compared to the conventional optical fiber. Furthermore, for a given MOF cladding geometry, the effective cladding index can have a strong dependence on the optical frequency, which enables the solid core MOFs to achieve interesting dispersion behaviors that can not be realized in conventional fiber geometry. ${ }^{7}$

Since both the solid core and the hollow core MOFs are typically composed of an array of air holes in a solid material (such as silica), they provide an ideal platform for the infiltration of liquid phase materials and the construction of microfluidic photonic devices. ${ }^{10-12}$ In most of the microfluidic photonic devices reported in the literature, all (or part) of the cladding air holes of the solid core MOF are filled with liquid. The presence of the liquid changes the effective cladding index of the composite MOF and consequently modifies the optical properties of light confined within the high index core. Based on this principle, microfluidic optical filters ${ }^{10,11}$ and switches ${ }^{12}$ have been realized.

In this paper, we present a microfluid-based fabrication procedure that allows us to realize functional MOFs through a selective filling technique. More specifically, we demonstrate a hybrid MOF in which only the central hollow core is filled with functional material whereas the cladding air holes remain intact. Therefore, the cladding structure of the functional MOF can still be treated using an effective index approximation. For a cladding structure with air filling fraction approaching 90\%, we can regard the fiber cladding as mostly air with an effective index of unity. As a result, we can achieve optical confinement in low index liquid (such as water) in such a functional MOF. Furthermore, the fiber core of the functional MOF can be almost any type of liquid phase material, and is no longer limited by the stringent requirement of the conventional fiber pulling process. It is clear that such a functional MOF is fundamentally 
different from the aforementioned microfluidic photonic devices, and may find wide applications in fields such as nonlinear optics, optical spectroscopy, chemical and biological sensing.

\section{FABRICATION}

The fabrication of the functional MOF begins with using a hollow core MOF as a template. Since optical confinement in the final functional MOF is provided by effective index guiding, we only require that the cladding structure of the template MOF has a large air filling fraction. We notice that this condition is much less stringent as compared to that of hollow core Bragg confinement. In our example, the MOF template is a silica hollow-core triangular lattice photonic crystal fiber as shown in Fig. 1(a). The fiber is fabricated by Crystal Fibre (Denmark) using a stack and draw technique. The scanning electron microscope (SEM) images of the fiber cross-section are shown in Fig. 1(b) and 1(c). The diameters of the central hole and the cladding holes are 5.8 and $2.8 \mu \mathrm{m}$, respectively. From the SEM images, we estimate that the air filling fraction of the cladding structure is approximately $90 \%$. The hollow core MOF template supports bandgap guiding in the wavelength range of $1.1 \mu \mathrm{m}$ to $1.3 \mu \mathrm{m}$. However, as discussed above, the fact that the template MOF supports hollow core guiding is not important, as long as the air filling fraction of the cladding structure is large enough such that the effective index of the cladding layers is close to 1 .

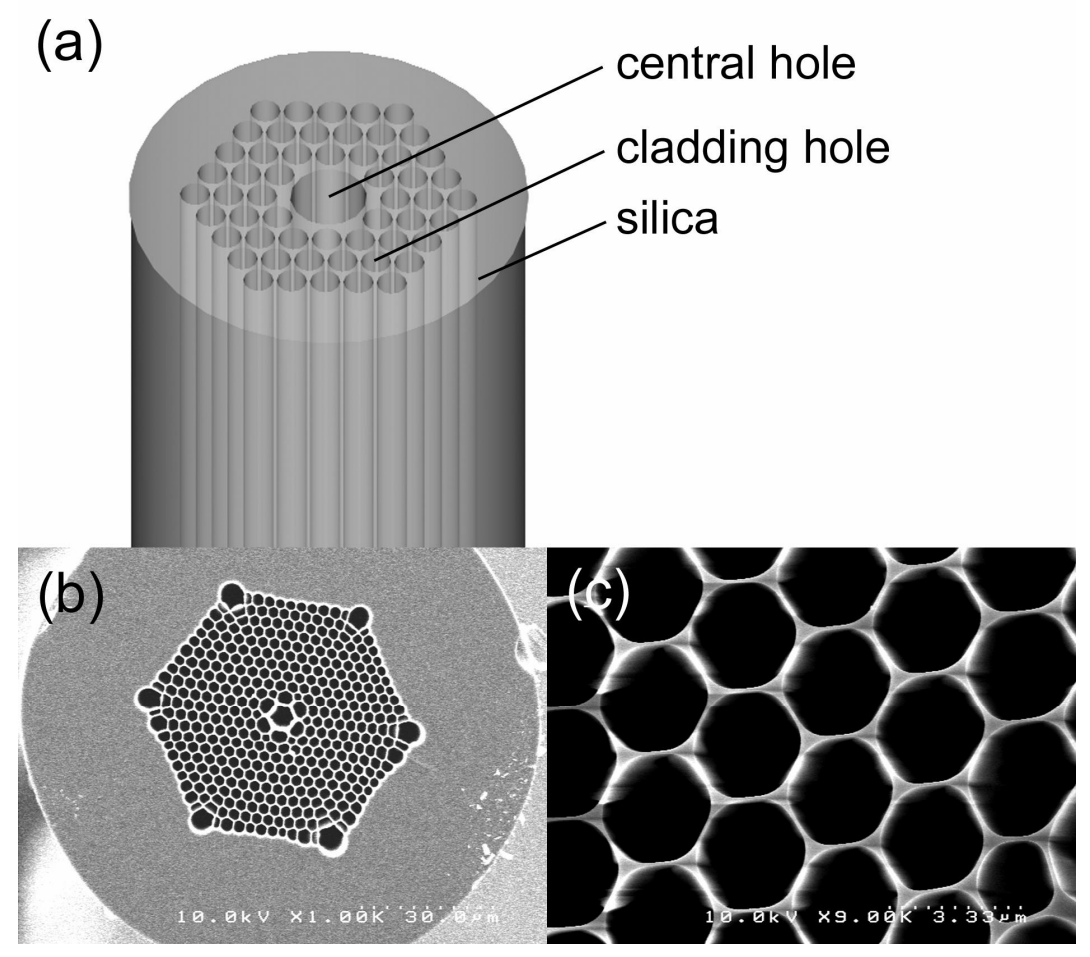

Fig. 1. Structure of the micro-structured optical fiber. Two differently sized holes are shown in the schematic diagram (a). The SEM images of the fiber cross-section show both the central hole and the cladding holes (b), as well as the triangular lattice of the cladding holes (c).

To fabricate the functional MOF, we begin by filling the silica MOF template with a UV curable polymer and curing the polymer. In Fig. 2, we show the SEM images of the cross-section of the MOF template filled with cured polymer. The fiber cross-section shown in Fig. 2 is close to the beginning of the filling point. At that position, we observe that all the air holes (including both the core and the cladding) are filled with polymer.

To selectively fill only the central air hole, we notice that the central core is significantly bigger than the surrounding cladding air holes. From fluid mechanics, we know that under constant pressure, the filling speed of a 
liquid in a cylindrical tube depends significantly on the tube size. Assuming laminar flow, the filling speed $v$ of the liquid in a hollow cylinder with radius $r$ can be written as: ${ }^{13}$

$$
v=-\frac{\Delta p r^{2}}{8 l \mu}
$$

where $\Delta p$ is the pressure difference between the two ends of the liquid tube within the cylindrical hole, $l$ is the infiltration length of the liquid within the hole, and $\mu$ is the viscosity of the liquid. Eq. (1) indicates that the liquid fills the larger air holes much faster than the smaller holes. Therefore, after a given period of time, the infiltration length of the polymer within the central hole is longer than that within the cladding holes.

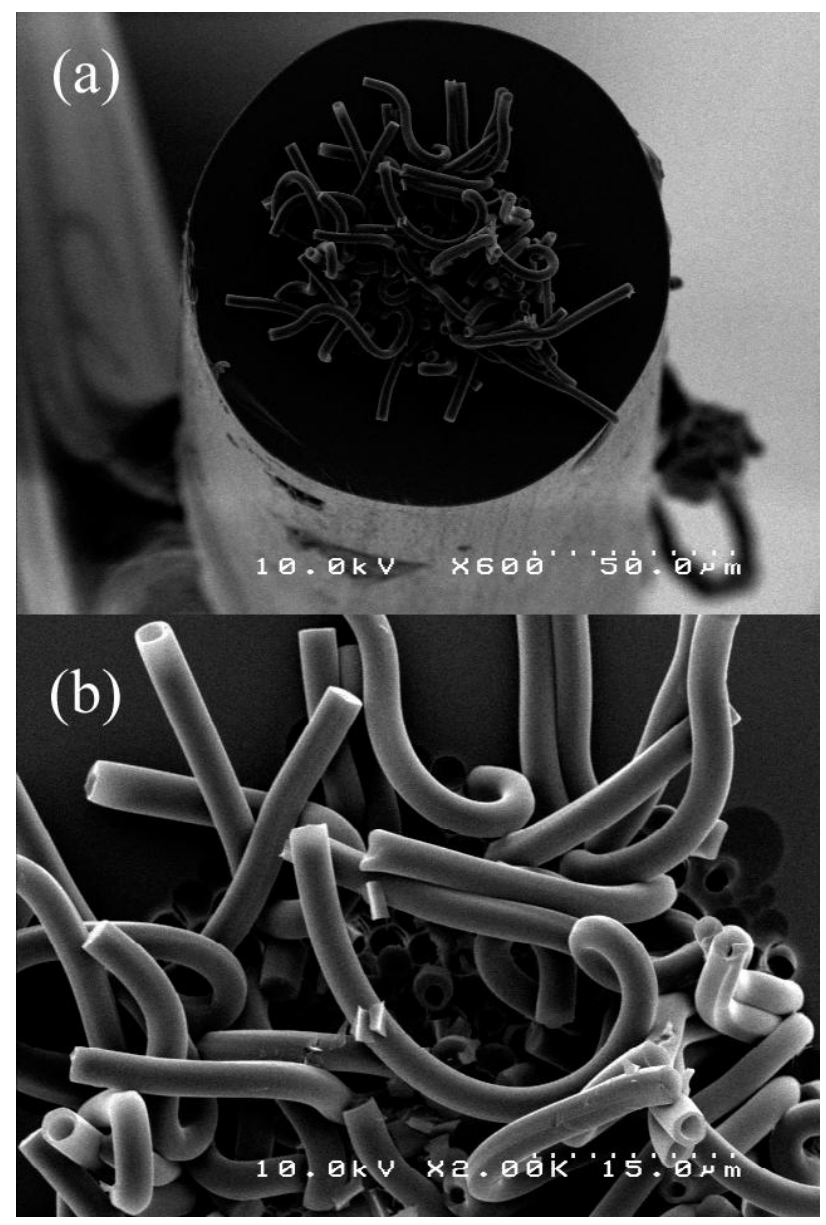

Fig. 2. SEM images of the cross-section of the silica MOF template filled with UV cured polymer.

In Fig. 3, we show several optical microscope images of cleaved cross-sections of the MOF template filled with UV cured polymer. Each image in Fig. 3 is labeled with a number, which refers to the distance between the cleaving position and the beginning point of polymer infiltration. From the figures, it is clear that close to the polymer infiltration point, both the cladding holes and the central hole are filled with UV cured polymer, as one might expect from Fig. 2. However, after a few centimeters $(2 \sim 4 \mathrm{~cm})$, only the central hole is filled polymer, whereas the rest of the cladding holes remains mostly empty. As a result, we can select filling either the central hole or the entire array of air holes by choosing an appropriate cleaving position. Based on this observation, we develop a selective filling technique that allows us to fill the big central core with almost any liquid phase material. The steps are outlined in Fig. 4. 


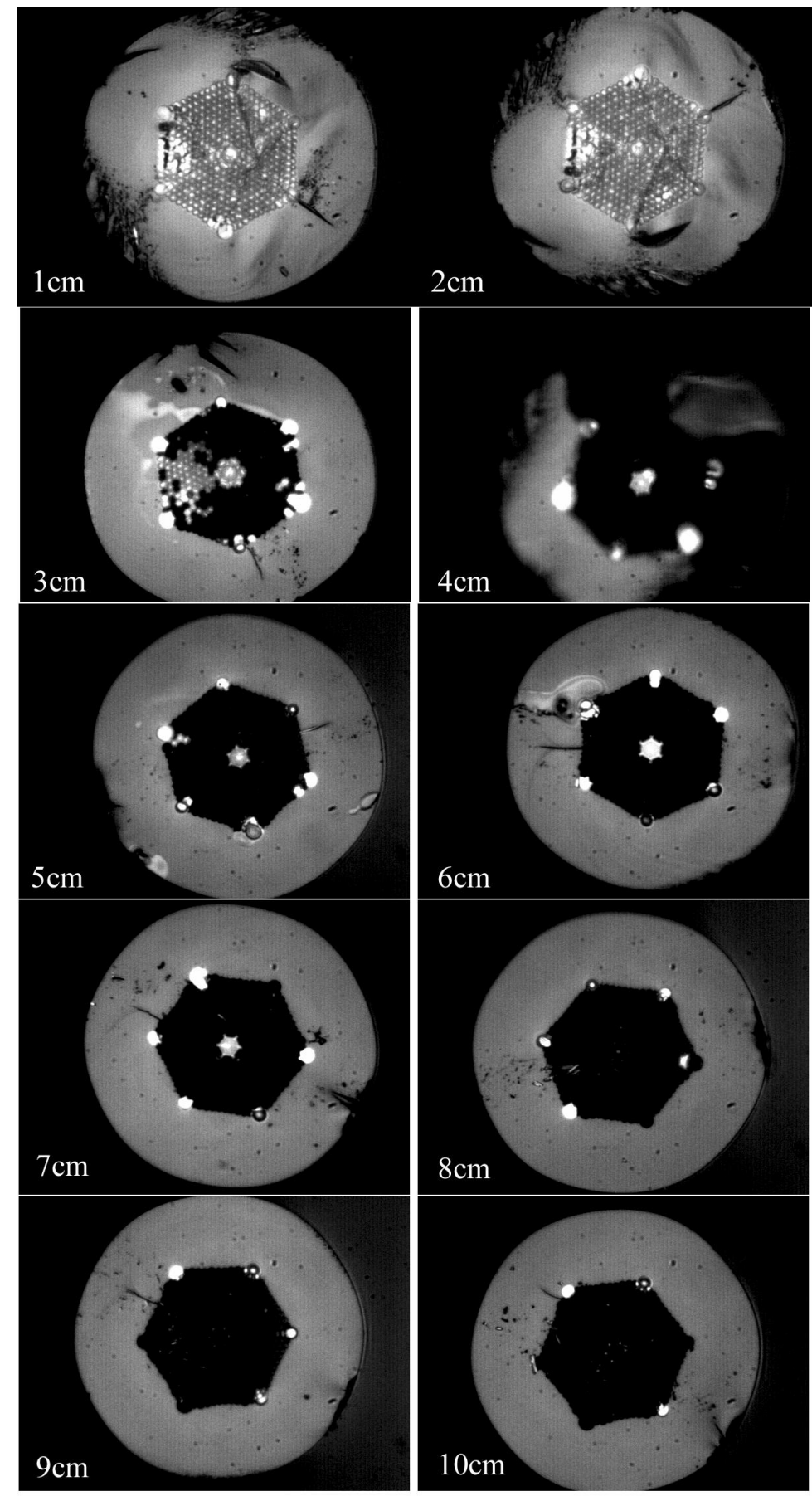

Fig. 3. Optical microscope images of the cross-section of the silica MOF template filled with polymer. The number in each image corresponds to the distance between the cleaving position and the beginning of polymer infiltration. 

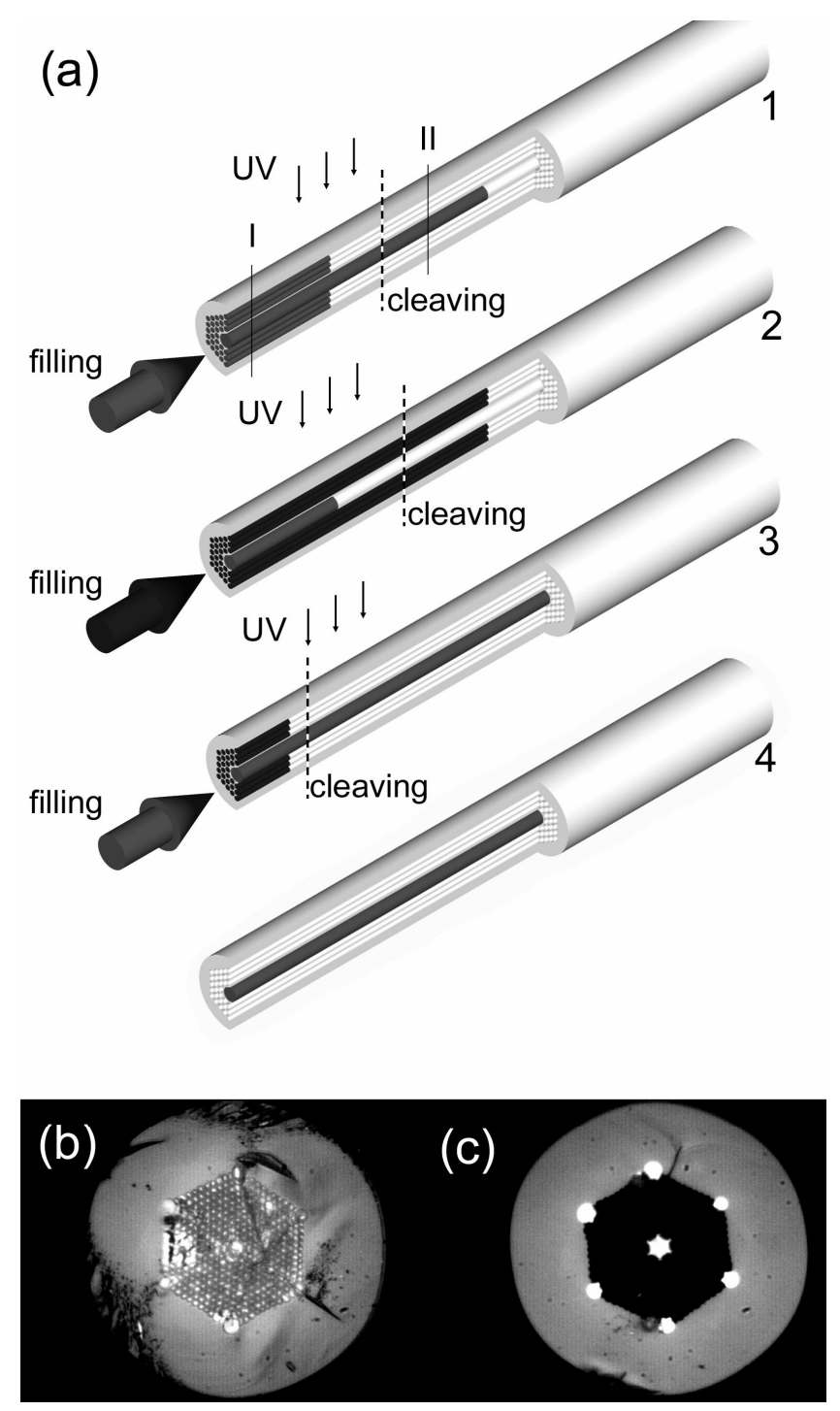

Fig. 4. Schematic flowchart on selective filling of the micro-structured optical fibers. The images (b) and (c) are optical microscope images of the fiber cross-section at the cleave positions I and II. The light region corresponds to the holes filled with polymer.

The fabrication process shown in Fig. 4(a) begins by using a syringe to pump a UV curable liquid phase polymer into the air holes of the MOF template. After a given period of time, the polymer infiltrates much deeper into the central air hole than the surrounding cladding holes (step 1). Along certain length from the input end of the MOF template, all the holes are filled with polymer [position I in step 1, Fig. 4(a)]. However, as demonstrated in Fig. 3, beyond that position, only the bigger holes are filled with polymer [position II in step 1, Fig. 4(a)]. The optical microscope images that correspond to position I and position II are given, respectively, in Fig. 4(b) and 4(c). Due to imperfect fabrication of the MOF template, we notice that in Fig. 4(c) the six holes around the edge of the cladding structure, which have sizes comparable to that of the central hole, are also filled with polymer. However, since these six holes are far from the central core region, filling them will not have any noticeable impact on the optical properties of the final functional MOF. We subsequently UV cure the polymer within the MOF and cleave the fiber at the position shown in step 1, which results in a modified MOF with the central hole capped by the cured polymer. With the presence of the cap, in step 2 we can inject any liquid or solution into the MOF cladding holes. The simplest choice is to use the same UV curable polymer. By applying pressure on the syringe, again we pump the polymer into the cladding holes and wait until the polymer penetrates beyond the length of the cap in the central hole. We subsequently UV cure the 
polymer and cleave the fiber at the position shown in step 2, Fig. 4(a). At the end of the second step, we have capped all the cladding holes with the cured polymer but left the central hollow core open. At this point, we have two choices. One approach is to directly pump liquid or solution-based material into the hybrid MOF core and keep the material within the core in liquid form. We will show later that such configuration has several distinctive advantages in spectroscopy and sensing applications. Alternatively, we can inject another type of curable liquid selectively into the central hole with the cladding holes plugged, and cure both ends of the fiber. After cleaving [as shown in Fig. 4(a), step 3], we obtain a hybrid MOF with the central hole filled with the desired functional material while the fiber cladding consists of an array of air holes in silica.

Compared with other types of MOFs, the hybrid MOF described above has several distinct advantages. For example, the core material of the functional MOF can be composed of almost any type of liquid-form materials, which allows a much broader range of possibilities as compared to the conventional step index fiber or MOF. Furthermore, since the functional MOF is not produced by the traditional fiber drawing tower, the choice of core material is not constrained by the stringent requirement of the fiber pulling process. One particularly interesting example of the core materials for the functional MOF is nonlinear optical polymer, which can have nonlinear coefficient several orders of magnitude higher than silica. We can also use dye molecules or quantum dots that are optically active in various wavelength ranges such as ultraviolet or visible. Consequently, we can construct optical fibers that are active in wavelength ranges not covered by the traditional silica-based fibers.

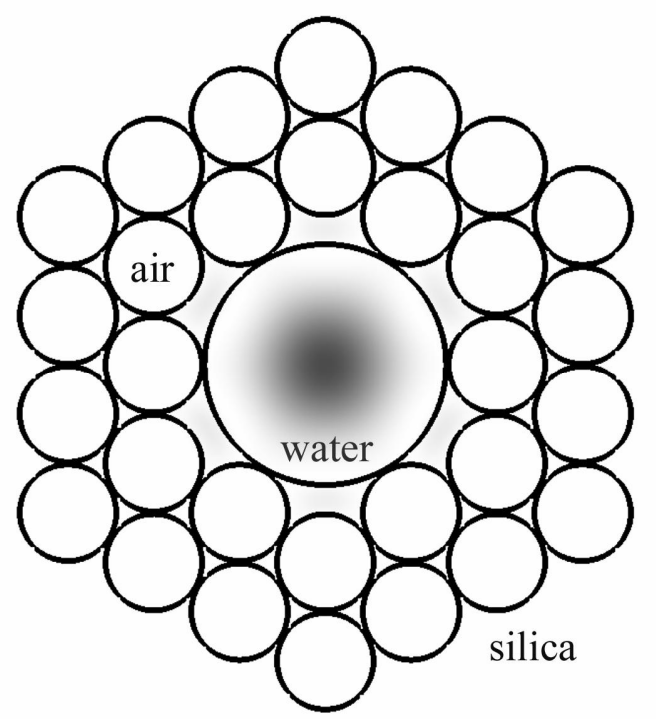

Fig. 5. The intensity profile of an optical mode propagating in a functional MOF with a water core. The cladding structure of the functional MOF consists of a triangular array of air holes in silica.

Another interesting case is a hybrid MOF with the cladding air filling fraction close to unity. In such a MOF, we can achieve optical guiding in low index materials such as water-based solution, which can potentially find important usage in chemical or biological sensing related applications. In Fig. 5, we show the profile of a guided mode in a hybrid MOF in which the central core is filled with water and the cladding structure is composed of a triangular lattice of air holes in silica. The modal profile is calculated using the multiple expansion method. ${ }^{14}$ Since the cladding effective index of the fiber is close to 1, the optical field is well confined within the low index water core, as can be seen from the figure. Consequently, we can achieve a long effective interaction length between light and the low index material, which is important for spectroscopy applications in water-based solutions. The only other optical fiber that can guide light in water-based solution core is the Teflon-AF coated capillary tube. ${ }^{15}$ However, compared with the Teflon coated fiber, the hybrid MOF fiber possesses several important advantages. First, silica-based fibers have been developed by the telecommunication industry for several decades. Therefore, low cost and high quality silica fibers are readily available. Furthermore, compared with Teflon coated capillary tube, hollow core silica fibers should have a much smoother inner surface, which leads to less surface scattering and lower loss. In addition, the cladding effective 
index of the hybrid MOF can be close to 1, which is significantly lower than the refractive index of Teflon. Consequently, the hybrid MOF can have a much larger numerical aperture, which leads to higher collection efficiency. The hybrid MOF can also have a much smaller core radius, which results in higher optical intensity and is important for nonlinear optical spectroscopy. Last but not least, compared with the Teflon coated fiber, the optical properties of a mode confined within the hybrid MOF can be much more precisely controlled by tuning the parameters of the core and cladding air holes of the silica MOF template.

\section{RESULTS}

We demonstrate an example of functional MOFs by following the selective filling technique described in section 2 and using Norland optical adhesive 73 (NOA73) as the UV curable polymer. The core material is UV cured NOA73 doped with 0.05 wt \% (0.0002 mol/L) Rhodamine 640 (R640). It should be mentioned that light in this MOF is confined by total internal reflection instead of Bragg reflection. The MOF can be handled in the same way as standard telecommunication fibers.
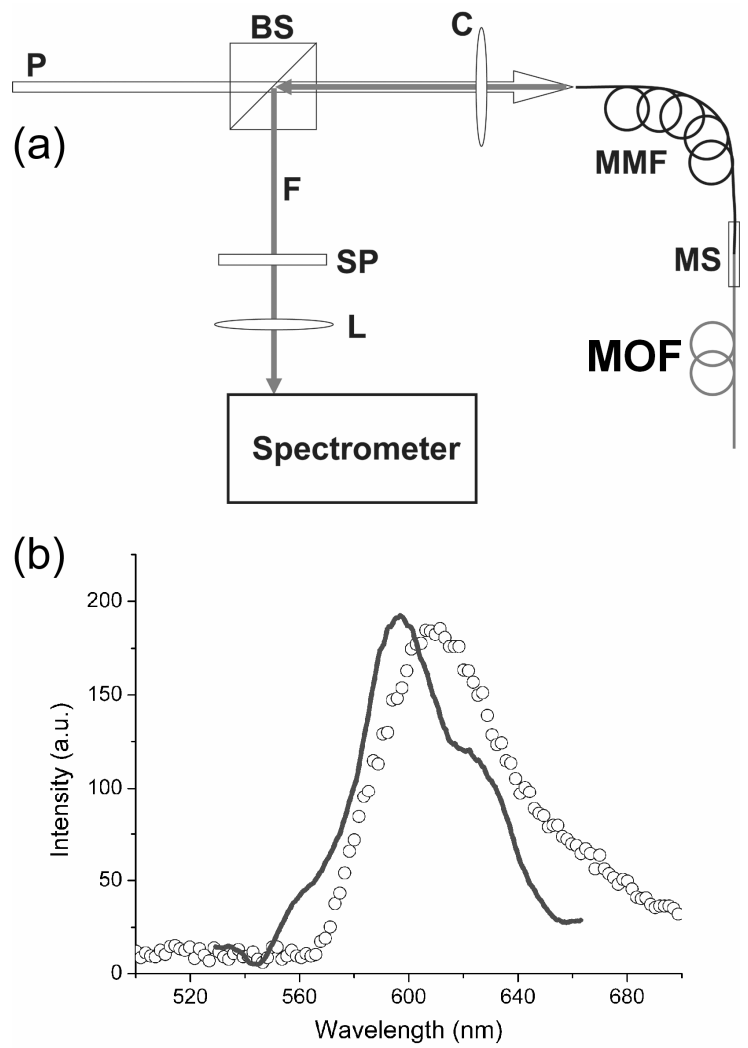

Fig. 6. Experimental setup for optical measurement of two-photon fluorescence from the functional MOF (a), P, pump; BS, beam splitter; C, collimator; MMF, multimode fiber; MS, mechanical splice; F, fluorescence; SP, short pass filter; L lens; MOF, functional micro-structured optical fiber. Detected fluorescence spectra (b) correspond to single photon (532 nm, open circle) and two-photon

(890 nm, solid line) pumping, respectively.

To measure the two-photon fluorescence from the functional MOF, we use the optical setup shown in Fig. 6(a). An 890-nm fs laser (200 fs, $76 \mathrm{MHz}, 15 \mathrm{~mW}$ ) is used as the pump. The pump light transmits through a beam splitter and is coupled into a multimode silica fiber. The multimode fiber is directly connected to the functional MOF via a mechanical splice. The back-traveling two-photon fluorescence is collected by the multimode fiber, and is measured by the spectrometer. The fluorescence spectrum of the hybrid MOF is shown in Fig 6(b). Compared with the conventional method, ${ }^{16,17}$ our demonstration provides a unique method for fabricating highly efficient two-photon fluorescence fiber devices. 


\section{CONCLUSIONS}

In summary, we propose and develop a method that allows the selective filling of MOFs with various functional materials. In particular, the selective-filling technique allows for the construction of a hybrid MOF in which the cladding structure is nearly all air and the central light-guiding core can be made from almost any solution-based materials. Using such a hybrid MOF, we can achieve optical confinement in low index materials such as water, which is of particular importance for spectroscopy and sensing applications in water-based solutions.

\section{ACKNOWLEDGEMENTS}

The authors wish to thank Prof. S. R. Quake, Dr. R. K. Lee, J. K. S. Poon, J. M. Choi, W. M. J. Green, W. Liang, and G. T. Paloczi for fruitful discussion and kind help. This work is supported by the National Science Foundation and Defense Advanced Research Projects Agency (Dr. D. Honey and D. R. Athale).

\section{REFERENCES}

1. P. Yeh, A. Yariv, and E. Marom, J. Opt. Soc. Am. 68, 1196 (1978).

2. C. M. Smith, N. Venkataraman, M. T. Gallagher, D. Muller, J. A. West, N. F. Borrelli, D. C. Allan, and K. W. Koch, Nature 424, 657 (2003).

3. F. Benabid, J. C. Knight, G. Antonopoulos, and P. S. J. Russell, Science 298, 399 (2002).

4. P. Russell, Science 299, 358 (2003).

5. J. C. Knight, Nature 424, 847 (2003).

6. J. C. Knight, T. A. Birks, and P. S. J. Russell, Opt. Lett. 21, 1547 (1996).

7. T. A. Birks, J. C. Knight, and P. S. J. Russell, Opt. Lett. 22, 961 (1997).

8. J. Herrman, U. Griebner, N. Zhavoronkov, A. Husakou, D. Nickel, J. C. Knight, W. J. Wadsworth, P. S. J. Russell, and G. Korn, Phys. Rev. Lett. 88, 173901 (2002).

9. J. Zagari, A. Argyros, N. A. Issa, G. Barton, G. Henry, M. C. J. Large, L. Poladian, and M. A. van Eijkelenborg, Opt. Lett. 29, 828 (2004).

10. C. Kerbage, and B. J. Eggleton, Appl. Phys. Lett. 82, 1338 (2003).

11. P. Domachuk, H. C. Nguyen, B. J. Eggleton, M. Straub, and M. Gu, Appl. Phys. Lett. 84, 1838 (2004).

12. T. T. Larsen, A. Bjarklev, D. P. Hermann, and J. Broeng, Opt. Express 11, 2589 (2003).

13. R. H. Sabersky, A. J. Acosta, E. G. Hauptmann, and E. M. Gates, Fluid Flow, $4^{\text {th }}$ ed. (Prentice Hall, Upper Saddle River, NJ, 1999).

14. T. P. White, B. T. Kuhlmey, R. C. McPhedran, D. Maystre, G. Renversez, C. M. de Sterke, and L. C. Botten, J. Opt. Soc. Am. B 19, 2322 (2002).

15. Z. Liu, and J. Pawliszyn, Anal. Chem. 75, 4887 (2003).

16. G. S. He, J. D. Bhawalkar, C. F. Zhao, C. K. Park, and P. N. Parasad, Appl. Phys. Lett. 68, 3549 (1996).

17. G. Jordan, T. Kobayashi, W. J. Blau, S. Pfeiffer, and H.-H. Horhold, Adv. Funct. Mater. 13, 751 (2003).

*Current address: Yong Xu, 302 Whittemore Hall, Department of Electrical and Computer Engineering, Virginia Tech, Blacksburg, VA 24061. Email: yong@ vt.edu. 